\title{
Overnight protection by inhaled salmeterol on exercise-induced asthma in children
}

\author{
K-H. Carlsen*+, O. Røksund+, K. Olsholt**, F. Njå+, J. Leegaard**, G. Bratten++
}

Overnight protection by inhaled salmeterol on exercise-induced asthma in children. $K-H$. Carlsen, O. Røksund, K. Olsholt, F. Njå, J. Leegaard, G. Bratten. OERS Journals 1995.

ABSTRACT: The main aim of the present study was to evaluate whether inhaled salmeterol given in the evening protected against exercise-induced asthma the next morning.

Twenty three children (12 males and 11 females) with a mean age of 11 yrs and with exercise-induced asthma participated in a double-blind, randomized, placebocontrolled study. The children inhaled salmeterol $25 \mu \mathrm{g}$, salmeterol $50 \mu \mathrm{g}$ and placebo by Diskhaler ${ }^{\circledR}$ at 10 p.m. on 3 separate days. Next morning, half of the children ran on a motor-driven treadmill for 6 min at submaximal load at 8 a.m. and the remainder at 10 a.m. Lung function was measured by maximal expiratory flowvolume loops before running, immediately after, and 3, 6, 10 and 15 min after running.

The mean maximum reduction in forced expiratory volume in one second (FEV1) after treadmill run was $34 \%$ before inclusion in the study. Mean maximum fall in FEV1 was significantly greater after placebo: $30 \%(23-36)(95 \%$ confidence interval) than after salmeterol $25 \mu \mathrm{g}$ : $19 \%(12-23)$ or salmeterol $50 \mu \mathrm{g}$ : $18 \%(12-25)$. In addition to the reduced postexercise bronchoconstriction, pre-exercise lung function (FEV1) was significantly higher both after salmeterol $25 \mu \mathrm{g}: 2.4 \mathrm{~L} \cdot \mathrm{s}^{-1}(\mathbf{2 . 1}-2.7)$ and salmeterol $50 \mu \mathrm{g}$ : $2.5 \mathrm{~L} \cdot \mathrm{s}^{-1}(2.2-2.8)$ than after placebo: $2.2 \mathrm{~L} \cdot \mathrm{s}^{-1}(1.9-2.5)$. No significant differences in pre- and postexercise lung function were found between children tested at 8 or 10 a.m., or in relation to salmeterol dosage.

Thus, inhaled salmeterol 25 and $50 \mu$ offered similar overnight protection against exercise-induced asthma and improved baseline lung function in the morning as compared to placebo.

Eur Respir J., 1995, 8, 1852-1855.
*Voksentoppen Center of Asthma and Allergy, Oslo, Norway. +Geilomo Children's Hospital of Asthma and Allergy, Geib, Norway. **Paediatric Department, Ullevål Hospital, Oslo, Norway. ${ }^{++}$Glaxo Norway AS

Correspondence: K-H. Carlsen

Voksentoppen Center of Asthma and Allergy Ullveien 14

$\mathrm{N}-0394$

Norway

\section{Keywords: Children}

exercise-induced asthma

placebo

salmeterol

treadmill run

Received: March 231995

Accepted after revision August 151995
Exercise-induced bronchoconstriction (EIB) is a common presentation of childhood asthma. Exercise-induced asthma (EIA) is defined by a reduction in lung function from before to after a standardized exercise manoeuvre. Forced expiratory volume in one second (FEV1) is the most common lung function measure employed, and a reduction in FEV1 of at least 10\%, (or 15\% according to some authors) from before to after exercise, is required to diagnose exercise-induced asthma [1]. In a random sample of Danish children and adolescents, $6 \%$ had a reduction in FEV1 of $15 \%$ or more after an exercise test, and $16 \%$ had a fall of at least $10 \%$ [2]. EIA often represents a major obstacle in the everyday life of asthmatic children [3]. As cardiovascular fitness has been found to correlate with psychological functioning of the asthmatic child, EIA may affect their self-image [4]. The physical activity of a child is often unpredictable, and is part of their regular play. It may, therefore, be difficult to employ the recommended use of inhaled $\beta_{2}$-agonists shortly before the exercise. A long-acting $\beta_{2}$-agonist might, thus, be beneficial in this respect. Salmeterol is a long-acting $\beta_{2}$-agonist for inhalation, and has been shown to protect against EIA in children [5] and in adults [6].
In the present study, we aimed to evaluate whether inhaled salmeterol taken the evening before, improved lung function and protected against exercise-induced asthma in the morning, 10-12 h after the administration of salmeterol.

\section{Patients and methods}

\section{Patients}

Twenty three patients with a history of EIA were included in the study, 11 boys and 12 girls, aged $8-16$ yrs. One additional boy dropped out during the run-in period due to acute gastroenteritis, which rendered him unable to fullfil the treadmill tests according to the schedule. Children with other severe diseases influencing cardiorespiratory function or performance on testing, were not eligible for the study. The regular asthma medication of the children included had to be unchanged during the last 4 weeks prior to the study, and the patients were not allowed to have had any lower airways infections during the 4 weeks prior to inclusion. 
Mean age of the patients was $11 \pm 2$ yrs (mean \pm standard deviation (SD)), weight was $42 \pm 12 \mathrm{~kg}$, and height was $148 \pm 13 \mathrm{~cm}$. Of the 23 children included in the study, 18 used inhaled steroids regularly in doses of 200-1000 $\mu \mathrm{g}$ b.i.d, 22 patients used inhaled short-acting $\beta_{2}$-agonists daily and two patients used antihistamines. Eleven of the 23 asthma patients had a history of atopic eczema, eight of allergic rhinitis, and one of food-allergy.

\section{Study design}

The present double-blind, randomized, cross-over study had a block design. Children participating in the study, who experienced a reduction in FEV1 of $15 \%$ or more after the treadmill test during the run-in visit, were included in the study. After inclusion into the study, they inhaled either placebo, salmeterol xinafoate $25 \mu \mathrm{g}$ or salmeterol xinafoate $50 \mu \mathrm{g}$ by Diskhaler $^{\circledR}$ at 10 p.m. A submaximal exercise test by running on a treadmill was performed the next morning at 8 or $10 \mathrm{a} . \mathrm{m}$. The patients were randomized into two main groups with half performing the treadmill test at 8 a.m. and half at 10 a.m. Within each main group they were randomized so that an equal number of patients received each drug as the first, second and third drug, respectively, employing a Greek-Latin square design. The design was set up for 24 patients. The interval between the study days was 2-7 days, except for three patients with an interval of 7-14 days. Sixteen patients were included in the study at Geilomo hospital, and seven patients at Ullevål Hospital. The study was approved by the Regional Medical Ethics Committee.

\section{Methods}

A single dose of salmeterol xinafoate $25 \mu \mathrm{g}$ dry powder, salmeterol xinafoate $50 \mu \mathrm{g}$ dry powder, or placebo dry powder packed in 8-place gold-foil Rotadisks ${ }^{\circledR}$ was inhaled by a Diskhaler ${ }^{\circledR}$ at 10.00 p.m. the night before each of the three study days. A proper inhalation technique with the Diskhaler ${ }^{\circledR}$ was ensured before including the patients in the study.

Lung function was measured by maximally forced expiratory flow-volume curves (Masterscreen Pneumo, Erich Jaeger GmbH \& Co. KG, Würzburg, Germany). EIB was determined by running on a motor-driven treadmill for 6 min with submaximal exercise load [7]. The inclination of the treadmill was $5.5 \%$, and the speed was adjusted to submaximal load to achieve a steady-state heart rate of 170-180 beats. $\mathrm{min}^{-1}$. The heart rate was recorded electronically (SportTester PE 3000 with memory function). The submaximal run on the treadmill was performed with the same speed (exercise load) on all four test occasions for the individual child. FEV1 was measured before running, immediately after, and 3, 6, 10 and 15 min after cessation of running.

Maximum percentage fall in FEV1 after the exercise test was calculated by: (pre-exercise FEV1 - minimum postexercise $\mathrm{FEV} 1$ )/pre-exerciseFEV $1 \times 100 \%$. Minimum postexercise FEV1 was taken from the recordings at 0, 3, 6, 10 and $15 \mathrm{~min}$ after the exercise test. Similar calculations were also performed for the lung function parameters: maximum expiratory flow at $50 \%$ of vital capacity (MEF50) and maximum expiratory flow at $25 \%$ of remaining vital capacity (MEF25)
The patients did not inhale short-acting $\beta_{2}$-agonist during the last $24 \mathrm{~h}$ before the treadmill tests. No patients used theophyllines or disodium cromoglycate. Antihistamines were stopped during the last week prior to the study. The use of inhaled steroids was not changed, but the patients did not receive their regular dose on the morning of the treadmill tests.

\section{Sample size}

With a power of $95 \%$ of detecting a $5 \%$ difference between the treatment groups for reduction in FEV1 after treadmill test at a significance level of $0.05,24$ patients should be included in the study [8].

\section{Statistical evaluation}

Results are given as mean values with $95 \%$ confidence intervals (CI) unless otherwise stated. Demographic data are given as mean \pm standard deviation (SD). Baseline data between the two blocks (treadmill at 8.00 and 10 a.m.) were compared by the nonparametric Wilcoxon rank sum test. When testing for differences between the two active drugs and placebo, analysis of variance (ANOVA) for mixed models was applied. When significant differences were found, pairwise comparisons were made by use of the Tukey method. Differences in number of patients with a fall in FEV $1>15 \%$ with the different treatments were tested with the chi-squared test. The analysis was performed with Statistical Analysis System (SAS) software. All tests were carried out with two tails and a significance level of $5 \%$.

\section{Results}

Pre-exercise lung function as well as postexercise reduction in FEV1, MEF50 and MEF25 did not differ significantly whether the exercise test was performed at 8.00 or 10.00 a.m. within each treatment group (placebo, and salmeterol 25 or $50 \mu \mathrm{g}$ ) (table 1). The mean postexercise

Table 1. - Lung function ( $\left.F E V_{1}\right)$, maximum reduction in $\mathrm{FEV}_{1}\left(\triangle \mathrm{FEV} \mathrm{V}_{1}\right)$ and maximum heart rate after treadmill run at 8.00 and 10.00 a.m. in 23 patients

\begin{tabular}{|c|c|c|c|}
\hline & 8.00 a.m. & 10.00 a.m. & p-value \\
\hline \multicolumn{4}{|l|}{ Run-in } \\
\hline FEV $1 L \cdot s^{-1}$ & $2.3 \pm 0.70$ & $2.3 \pm 0.78$ & NS \\
\hline$\triangle \mathrm{FEV}_{1} \%$ & $34 \pm 16$ & $32 \pm 12$ & NS \\
\hline HR beats $\cdot \mathrm{min}^{-1}$ & $188 \pm 9$ & $187 \pm 9$ & NS \\
\hline \multicolumn{4}{|l|}{ Placebo } \\
\hline FEV1 $\quad L \cdot s^{-1}$ & $2.2 \pm 0.77$ & $2.2 \pm 0.71$ & NS \\
\hline$\triangle \mathrm{FEV}_{1} \%$ & $33 \pm 15$ & $29 \pm 14$ & NS \\
\hline HR beats $\cdot \mathrm{min}^{-1}$ & $184 \pm 8$ & $183 \pm 7$ & NS \\
\hline \multicolumn{4}{|l|}{ Salmeterol $25 \mu \mathrm{g}$} \\
\hline $\mathrm{FEV}_{1} \mathrm{~L} \cdot \mathrm{s}^{-1}$ & $2.5 \pm 0.73$ & $2.4 \pm 0.74$ & NS \\
\hline$\triangle \mathrm{FEV}_{1} \%$ & $24 \pm 19$ & $15 \pm 11$ & NS \\
\hline HR beats $\cdot \min ^{-1}$ & $186 \pm 8$ & $179 \pm 6$ & NS \\
\hline \multicolumn{4}{|l|}{ Salmeterol $50 \mu \mathrm{g}$} \\
\hline FEV1 L·s ${ }^{-1}$ & $2.5 \pm 0.78$ & $2.5 \pm 0.82$ & NS \\
\hline$\triangle \mathrm{FEV}_{1} \%$ & $23 \pm 16$ & $14 \pm 13$ & NS \\
\hline $\mathrm{HR}$ beats $\cdot \mathrm{min}^{-1}$ & $185 \pm 7$ & $181 \pm 8$ & NS \\
\hline
\end{tabular}

Data are presented as mean \pm SD. FEV1: forced expiratory volume in one second; HR: heart rate; NS: nonsignificant. 

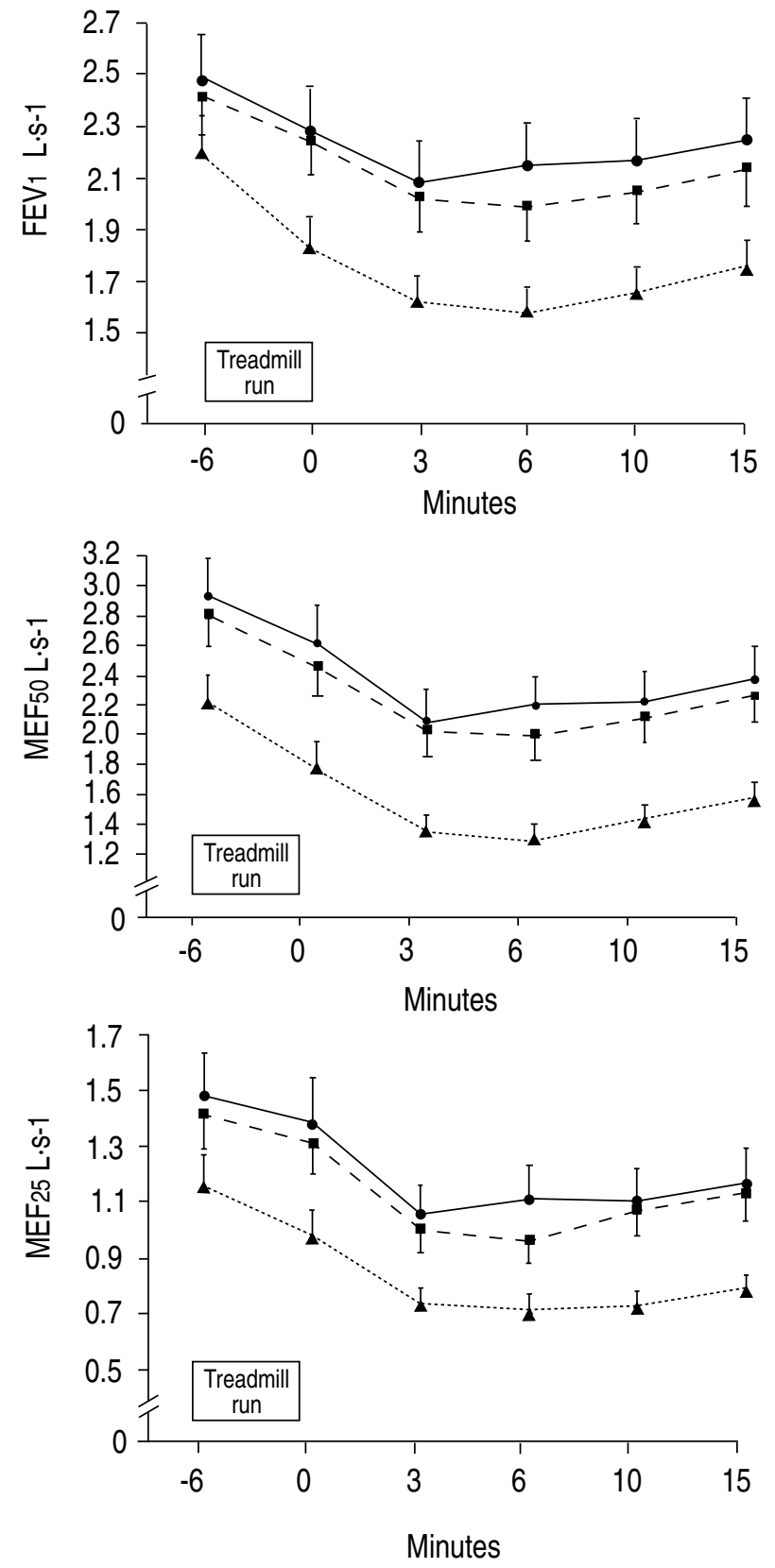

Fig. 1. - FEV1, MEF50 and MEF25 pre-exercise, immediately postexercise, and 3, 6, 10 and 15 min after treadmill run. The treadmill tests were performed the morning after placebo, salmeterol 25 and $50 \mu \mathrm{g}$ were inhaled at 10 p.m. There were significant differences between placebo and both doses ( 25 and $50 \mu \mathrm{g}$ ) of salmeterol both pre-exercise and on all measurements after exercise ( $p<0.0005$ on all comparisons). No significant differences were found between salmeterol 25 and $50 \mu \mathrm{g}$. Values are presented as mean and SEM. ——: salmeterol $50 \mu \mathrm{g}$; - - - : : salmeterol $25 \mu \mathrm{g} ; \cdots . . . . \cdots \cdots . . .$. : placebo. FEV1: forced expiratory volume in one second; MEF50: maximum expiratory flow at 50\% vital capacity; MEF25: maximum expiratory flow at $25 \%$ remaining vital capacity

heart rate was not significantly different at 8.00 and 10.00 a.m. Further evaluation was, therefore, performed with the results from 8 and 10 a.m. analysed together for each treatment.

The values of FEV1, MEF50 and MEF25 before and immediately after exercise, and 3, 6, 10 and 15 min after exercise are shown in figure 1. Significant differences
Table 2. - FEV 1 , maximum reduction in $\mathrm{FEV}_{1}\left(\Delta \mathrm{FEV} \mathrm{V}_{1}\right)$ after exercise, number of patients (Pts) with $>15 \%$ reduction in FEV 1 after exercise and heart rate immediately postexercise on the three treatment days in 23 patients.

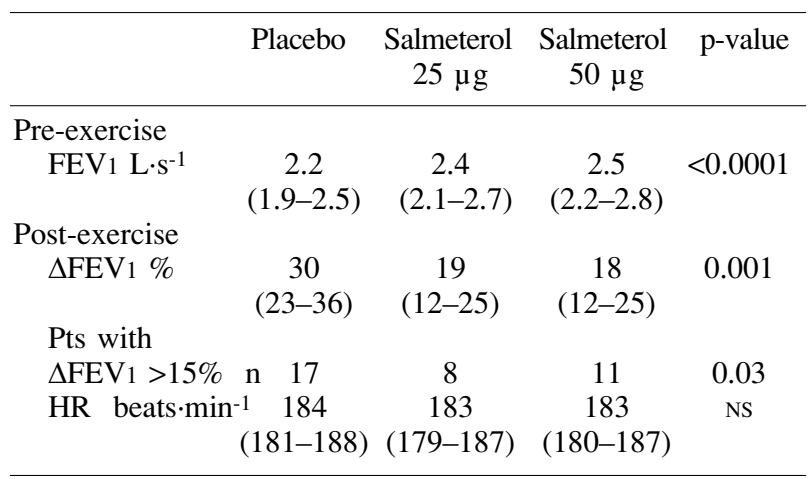

Values are presented as mean with $95 \%$ confidence interval in parenthesis. Numbers of patients are in absolute numbers. Pvalues represent differences between placebo and the two doses of salmeterol. No differences were found between salmeterol 25 and $50 \mu \mathrm{g}$. For abbreviations see legend to table 1 .

were found between placebo and salmeterol $25 \mu \mathrm{g}$ on the one hand, and between placebo and salmeterol $50 \mu \mathrm{g}$ on the other both in pre-exercise values of FEV1, MEF50 and MEF25: $(\mathrm{p}<0.0001)$ and after the treadmill test $(\mathrm{FEV} 1$ and MEF50: $p<0.0001$; MEF25: $p<0.0005)$. No significant differences were found between the lung function values pre-exercise or postexercise for salmeterol $25 \mu \mathrm{g}$ and $50 \mu \mathrm{g}$. The mean values with standard error of the mean (SEM) for FEV1, MEF50 and MEF25 before and after the exercise tests are shown in figure 1, demonstrating the bronchodilator effect of both doses of salmeterol preexercise, as well as the higher lung function values after both salmeterol doses compared to placebo through the entire observation period after the exercise tests.

The reduction in FEV1 was significantly greater $(p=0.02)$ after placebo treatment (mean value $0.67 \mathrm{~L} \cdot \mathrm{s}^{-1}$ ) than after salmeterol $25 \mu \mathrm{g}\left(0.47 \mathrm{~L} \cdot \mathrm{s}^{-1}\right)$ or $50 \mu \mathrm{g}\left(0.46 \mathrm{~L} \cdot \mathrm{s}^{-1}\right)$, as seen in table 2. The percentage reduction in FEV1 after exercise from pre-exercise value was also significantly greater $(\mathrm{p}=0.001)$ after placebo treatment $(30 \%)$ than after salmeterol $25 \mu \mathrm{g}(19 \%)$ and salmeterol $50 \mu \mathrm{g}(18 \%)$ (table 2). No such significant differences were found between the two doses of salmeterol.

Regarded as a measure of the exercise load of the treadmill tests, the heart rate immediately after the exercise did not differ significantly between placebo (184 beats $\left.\cdot \mathrm{min}^{-1}\right)$, salmeterol $25 \mu \mathrm{g}\left(183\right.$ beats $\left.\cdot \mathrm{min}^{-1}\right)$ and salmeterol $50 \mu \mathrm{g}\left(183\right.$ beats $\left.\cdot \mathrm{min}^{-1}\right)$ (table 2$)$.

The number of subjects with a maximum reduction in FEV1 more than $15 \%$ from pre-exercise level differed significantly $(\mathrm{p}=0.03)$ after placebo treatment (17 patients) compared to salmeterol $25 \mu \mathrm{g}$ (8 patients) and salmeterol $50 \mu \mathrm{g}$ (11 patients), but did not differ significantly between salmeterol 25 and $50 \mu \mathrm{g}$ (table 2).

\section{Discusssion}

The present study shows that inhaled salmeterol 25 and $50 \mu \mathrm{g}$ taken in the evening, significantly improves 
lung function the next morning, 10 and $12 \mathrm{~h}$ later. Furthermore, the fall in lung function after exercise was significantly diminished by salmeterol 25 and $50 \mu \mathrm{g}$ compared to placebo. This demonstrates a prolonged effect of the inhaled drug as regards bronchodilation, as well as protection against EIA.

GREEN and PRICE [5] have previously demonstrated a protection against EIA by salmeterol $50 \mu \mathrm{g}, 1,5$ and $9 \mathrm{~h}$ after administration of the drug. The present study demonstrated that the protective period of inhaled salmeterol may last 10 and $12 \mathrm{~h}$, and that a similar protection was offered by the lower dose of $25 \mu \mathrm{g}$ salmeterol.

The improved lung function the morning after inhalation of salmeterol at $10 \mathrm{p} . \mathrm{m}$. in the present study indicates that an amelioration of nocturnal asthma symptoms may be anticipated after inhaling salmeterol in the evening. This finding compares to the improvement in nocturnal symptoms and morning PEF obtained by inhaled salmeterol compared to slow-release terbutaline, recently reported [9].

The long-acting duration of salmeterol, both as regards improved lung function and protection against EIA, would probably help in improving the quality of life in asthma patients, as recently found in a study from general practice in England. The adult patients in that study experienced improved asthma symptoms in general, as well as an improvement in activity restriction [10]. Asthma limits physical activity to a great extent in asthmatic children [3, $11]$, and physical activity is an important part of their life [12]. To obtain protection against EIA which may last for up to $12 \mathrm{~h}$, would enable the asthmatic children to take part in normal play and sports. The common recommendation, to inhale a short-acting $\beta_{2}$-agonist or disodium cromoglycate shortly before physical activity, may be troublesome for a child. Addition of a long-acting $\beta_{2}$-agonist for inhalation to the regular anti-inflammatory therapy, may therefore be of benefit in many patients.

In the present study, the exercise tests were performed at 8 a.m. (10 h after adminstration of the study drug) in half of the patients, and at $10 \mathrm{a} . \mathrm{m}$. for the remainder. The results both for placebo, and for salmeterol 25 and $50 \mu \mathrm{g}$ did not differ signficantly between the block tested at 8 a.m. and the block tested at $10 \mathrm{a} . \mathrm{m}$. The two blocks were, therefore, evaluated together. However, when performing the analyses for each block, the results were the same with the same significant differences within each block as when the two blocks were evaluated together.

The exercise load was standardized in the present study, as in the study by BONER et al. [13] and in the study by GrEen and Price [5]. Boner et al. [13] employed a load of approximately $90 \%$ of predicted maximum value measured by heart rate, and this compares well to the load in the present study. The load was practically identical on all three tests in our study (table 2), thus making an adequate comparison between the different treatment days possible.

It has been maintained that inhaled $\beta_{2}$-agonists protect against EIA due to their bronchodilating effect, and that they should be used primarily for EIA when a bronchoconstriction may be demonstrated [14]. The present study confirms that the protective effect of salmeterol is caused, to a large extent, by its bronchodilating effect. The preexercise lung function was signficantly higher after both doses of salmeterol than after placebo. Furthermore, the higher post-exercise lung function after salmeterol was explained, to a large extent, but not fully, by the higher preexercise level (fig. 1 and table 2). Thus, in the present study, salmeterol protected against EIA mainly through its bronchodilating effect.

From this study, it might be observed that salmeterol did not fully protect against EIB. Eight subjects had a reduction in FEV1 greater than $15 \%$ after inhaled salmeterol 25 $\mu \mathrm{g}$, and 11 subjects after salmeterol $50 \mu \mathrm{g}$. Additional protective medication taken shortly before exercise, may be needed to fully control EIB in some patients. Thus, it is advisable to check the effect of the drug upon EIB before giving a long-term prescription.

In conclusion, the present study has demonstrated that inhaled salmeterol 50 and $25 \mu \mathrm{g}$ had a protective effect upon EIA. The protective effect was demonstrated to last for $12 \mathrm{~h}$ after inhaling salmeterol. Thus, inhaled salmeterol may help asthmatic children to improve their asthmatic symptoms and their control of EIB.

Acknowledgements: The authors thank L. Möhlenberg and M. Bru for technical help during the study.

\section{References}

1. Carlsen KH, Boe J. Exercise-induced asthma in children. Eur Respir J 1993; 6: 614-616.

2. Backer V, Ulrik CS. Bronchial responsiveness to exercise in a random sample of 494 children and adolescents from Copenhagen. Clin Exp Allergy 1992; 22: 741-747.

3. Taylor WR, Newacheck PW. Impact of childhood asthma upon health. Pediatrics 1992; 90: 657-662.

4. Strunk RS, Mrazek DA, Fukuhara JT, Masterson J, Ludwick SK, LaBrecque. Cardivascular fitness in children with asthma correlates with psychological functioning of the child. Pediatrics 1989; 84: 460-464.

5. Green CP, Price JF. Prevention of exercise induced asthma by inhaled salmeterol xinafoate. Arch Dis Child 1992; 67: 1014-1017.

6. Anderson SD, Rodwell LT, Du Toit J, Young IH. Duration of protection by inhaled salmeterol in exercise-induced asthma. Chest 1991; 100: 1254-1260.

7. Oseid S, Haaland K. Exercise studies on asthmatic children before and after regular training. In: Eriksson B, Furberg B, eds. Swimming Medicine. IV. Baltimore, University Park Press, 1978; pp. 32-41.

8. Larsen S. Sample size in controlled clinical trials. Scand J Gastroenterol 1985; 20: 395-400.

9. Brambilla C, Chastang C, Georges D, Bertin L. Salmeterol compared with slow-release terbutaline in nocturnal asthma. Allergy 1994; 49: 421-426.

10. Jones KP, UK study group. Salmeterol xinafoate in the treatment of mild to moderate asthma in primary care. Thorax 1994; 49: 971-975.

11. Lenney W, Well NEJ, O'Neill BA. The burden of paediatric asthma. Eur Respir Rev 1994; 4: 49-62.

12. Oseid S, Aas K. Exercise-induced asthma. Allergy 1978; 33: 227-228.

13. Boner AL, Spezia E, Piovesan P, Chiocca E, Maiocchi G. Inhaled formoterol in the prevention of exerciseinduced bronchoconstriction in asthmatic children. Am J Respir Crit Care Med 1994; 149: 935-939.

14. Anderson SD. Drugs and the control of exercise-induced asthma. Eur Respir J 1993; 6: 1090-1092. 